\title{
Nonaxial-octupole effect in superheavy nuclei
}

\author{
Y.-S. Chen ${ }^{1}$, Yang Sun $^{2,3}$, Zao-Chun Gao ${ }^{1,4}$ \\ ${ }^{1}$ China Institute of Atomic Energy, P.O. Box 275(18), Beijing 102413, People's Republic of China \\ ${ }^{2}$ Department of Physics, Shanghai Jiao Tong University, Shanghai 200240, People's Republic of China \\ ${ }^{3}$ Joint Institute for Nuclear Astrophysics, University of Notre Dame, Notre Dame, Indiana 46556, USA \\ ${ }^{4}$ Department of Physics, Central Michigan University, Mount Pleasant, Michigan 48859, USA
}

(Dated: October 30, 2018)

\begin{abstract}
The triaxial-octupole $\mathrm{Y}_{32}$ correlation in atomic nuclei has long been expected to exist but experimental evidence has not been clear. We find, in order to explain the very low-lying $2^{-}$bands in the transfermium mass region, that this exotic effect may manifest itself in superheavy elements. Favorable conditions for producing triaxial-octupole correlations are shown to be present in the deformed single-particle spectrum, which is further supported by quantitative Reflection Asymmetric Shell Model calculations. It is predicted that the strong nonaxial-octupole effect may persist up to the element 108. Our result thus represents the first concrete example of spontaneous breaking of both axial and reflection symmetries in the heaviest nuclear systems.
\end{abstract}

PACS numbers: 21.10.Re, 21.60.Cs, 27.90.+b

The issue that underlies the present investigation is whether some heaviest atomic nuclei can be characterized as having an intrinsic mean field with nonaxial-octupole component, in connection with the tetrahedral symmetry which is quite common in molecular, metallic clusters, and some other quantum objects. The tetrahedral symmetry is a direct consequence of the point group and corresponds to the invariance under transformation of the group $\mathrm{T}_{d}^{D}$ which has two one- and one four-dimensional irreducible representation. In molecular and metallic clusters, the tetrahedral symmetry is determined by mutual geometric arrangement of the constituent ions. In atomic nuclei, which are finite many-body systems with the strong interaction, the situation is more complex. However, it is usually understood that nuclear shapes are governed by the shell effects.The tetrahedral instability in finite many-fermion systems was suggested in 1991 [1]. Tetrahedral shape in nuclei was predicted with more realistic model calculations $[2,3,4,5]$.

For description of nuclear shape, it has been proven to be extremely useful to parameterize the surface $R(\theta, \phi)$ through its expansion in the spherical harmonic function $Y_{\lambda \mu}^{*}(\theta, \phi)$, where $\theta$ and $\phi$ are the rotation angles [6]. The coefficients of the expansion, $\alpha_{\lambda \mu}$, are related to deformation parameters. The quadrupole deformation $(\lambda=2)$ is the leading deformation effect known almost for all the nuclei throughout the nuclear chart. The next one, the octupole deformation $(\lambda=3)$, is related to a spontaneous breaking of reflection symmetry, or parity, and is known in some nuclei. The tetrahedral symmetry is realized at the first order through the nonaxial-octupole $\mathrm{Y}_{32}$ deformation [7, 8]. Almost pure $Y_{32}$ shape was suggested by the two level model study of finite Fermion systems[9]. Calculations of possible nonaxial pear-like ground-state deformations in the mass region $220<A<230$ has been reported [10]. Both axially- and nonaxially-enhanced octupole correlations have been suggested for the $A=90$ mass region [11] and in the superdeformed Hg nuclei [12]. The Skyrme HFB calculation has predicted that some nuclei can be extremely soft against the $Y_{32}$ tetrahedral deformation [13, 14].
One may thus conclude that there have been many theoretical discussions about this exotic type of nuclear deformation; however, experimental evidence has not been very clear. This may imply that the tetrahedral symmetry and the pure $\mathrm{Y}_{32}$ shape manifest themselves in nuclear physics in a very complex way. The octupole mode, as a smaller deformation effect, has to compete with the dominant quadrupole mode, and as a result, the appearance of the tetrahedral symmetry in all known mass regions is somewhat obscure. The question is whether one can find evidences in the heaviest mass region which is still less known. It is legitimate to ask this question because a strong octupole effect has been well known to exist in the actinide region [15] which is the closest neighboring mass region to the heaviest nuclei. The present study will try to answer this question. Some experimental data, though very limited and incomplete, have already indicated such a possibility. These data show that very low-lying negative parity bands with the sequence of $I^{\pi}=2^{-}, 3^{-}, 4^{-}, 5^{-}, \cdots$ appear in the spectrum of some very heavy nuclei (see discussions below).

Exploring the predicted stable island of superheavy elements is the current goal in nuclear science. In the past few years, progress has been made in synthesis of new elements (for review, see Refs. [16, 17, 18, 19]). On the other hand, the heaviest nuclei for which detailed spectroscopic measurements can be performed are the isotopes with proton number $Z \sim 100$ and neutron number $N \sim 150[20,21,22]$. The significance of studying the deformed nuclei in this transfermium region is that it can provide an indirect way to access the single-particle states of the next spherical shells because some of these orbitals are strongly down-sloping, and thus can come close to the Fermi surface in the deformed region [23].

There has been experimental observation of low-energy $2^{-}$ bands in the mass-250 nuclei. For example, for the $N=150$ isotonic chain, early data showed a $2^{-}$band in ${ }^{246} \mathrm{Cm}$ with an bandhead energy $E\left(2^{-}\right)=0.842 \mathrm{MeV}$ [26], and in ${ }^{248} \mathrm{Cf}$ with a much lower bandhead $E\left(2^{-}\right)=0.592 \mathrm{MeV}$ [27]. The recent data have added new evidences for ${ }^{250} \mathrm{Fm}$ with $E\left(2^{-}\right)=0.879$ 


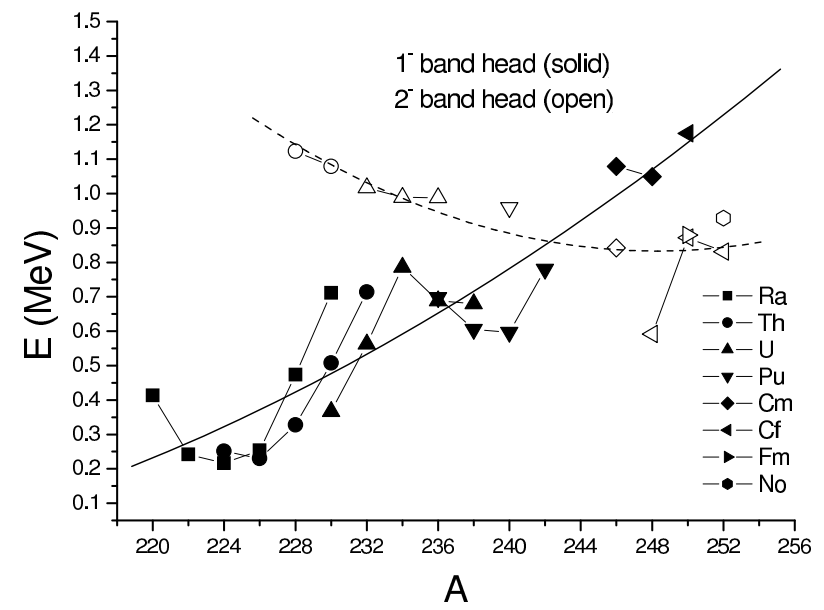

FIG. 1: Experimental energies of the $1^{-}$(solid symbols) and $2^{-}$ (open symbols) bandheads of the isotopes $\mathrm{Ra}, \mathrm{Th}, \mathrm{U}, \mathrm{Pu}, \mathrm{Cm}, \mathrm{Cf}$, $\mathrm{Fm}$, and No. Data for $\mathrm{Ra}, \mathrm{Th}, \mathrm{U}$, and $\mathrm{Pu}$ are taken from Refs. [24, 25], and for Cm, Cf, Fm, and No from Refs. [26, 27, 28, 29]. The solid line and dash line are the polynomial fits for the $1^{-}$and $2^{-}$bandhead energies, respectively. Note that the two curves cross at $A=242$.

$\mathrm{MeV}$ [28] and for ${ }^{252} \mathrm{No}$ with $E\left(2^{-}\right)=0.929 \mathrm{MeV}$ [29]. We find it difficult to interpret these bands as 2-quasiparticle (qp) excitations in a reflection-symmetric well because of their low bandhead energy, particularly for ${ }^{248} \mathrm{Cf}$. The reason is simple. To be excited, a 2-qp state must have an energy greater than two times of the BCS pairing gap, and the pairing gaps in this mass region are typically $\sim 0.5 \mathrm{MeV}$ for neutrons and $\sim 0.8 \mathrm{MeV}$ for protons, which are the needed amount to correctly reproduce the rotational behavior, e.g. the moment of inertia. Therefore, any (relatively) pure 2-qp state should lie higher than $1 \mathrm{MeV}$ above the ground state. For the observed low-energy $2^{-}$bands, there must be strong correlations that sensitively act on the negative-parity configurations to push them down. The primary candidate of the correlations is of the octupole type.

The purpose of the present article is to investigate the role of the nonaxial-octupole correlations in collective excitations of transfermium and superheavy nuclei. We first analyze the systematical trend of experimental low-lying spectroscopy data, associated with axial-octupole and nonaxial-octupole modes and ranging from the $A=220$ (actinide) to the 250 (transfermium) mass region. This analysis convincingly shows the importance of introducing nonaxial-octupole correlations in the description of low-lying $2^{-}$bands in the $A=250$ mass region. Second, we generalize the Reflection Asymmetric Shell Model (RASM) [33] to include the triaxial-octupole degree of freedom, which is applied to describe quantitatively the $2^{-}$ bands. Based on these results, we interpret these bands in the heaviest nuclear systems as having connection to the predicted tetrahedral symmetry.

Experimental energies of the lowest $1^{-}$and lowest $2^{-}$ bandheads of Ra, Th, U, Pu, Cm, Cf, Fm, and No are plotted as functions of mass number in Fig. 1. To qualify a "band",

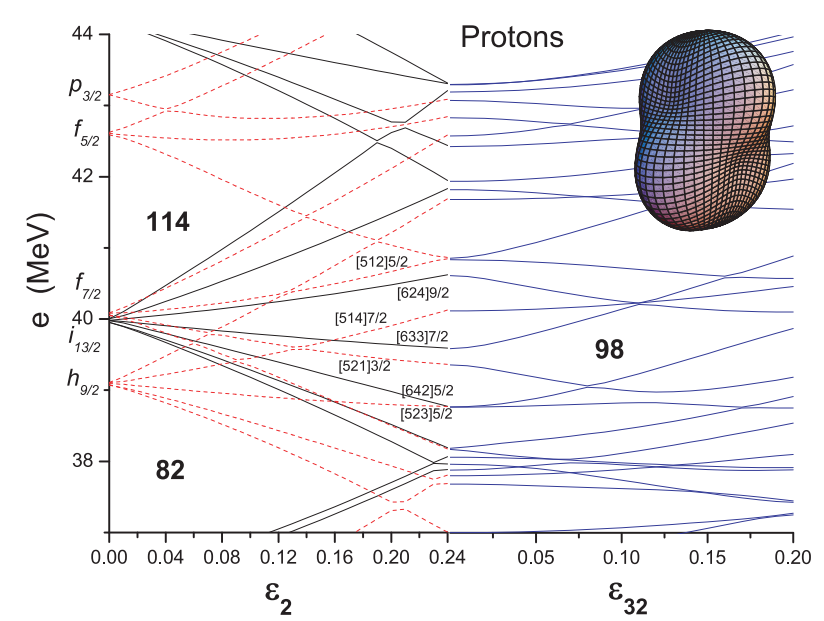

FIG. 2: (Color online) Proton Nilsson diagram showing singleparticle orbitals as deformation $\varepsilon_{2}$ varies (left part), and as functions of $\varepsilon_{32}$ with fixed $\varepsilon_{2}=0.24$ (right part). In the left part, orbitals with positive (negative) parity are shown by solid (dashed) curves.

we use a criterion which requires that for each band, at least three lowest states be assigned experimentally. As a general tendency, it is seen from Fig. 1 that the $1^{-}$bandhead energies increase with increasing mass number, while the $2^{-}$ones behave like a gradually decreasing function of mass number. In the Ra-Pu region, the $1^{-}$bands lie very low in energy while the $2^{-}$bands are quite high. This reflects the well-known fact that in Ra-Pu nuclei, the axial-octupole correlation, associated with the $\mathrm{Y}_{30}$ shape, is important. However, the situation becomes opposite in the Cm-Cf-Fm-No region where the $2^{-}$ bandhead energies drop to lower values. The occurrence of low-lying $2^{-}$bands indicates that the nonaxial-octupole effect becomes important in the transfermium region.

The polynomial fits for the $1^{-}$(solid curve) and $2^{-}$(dash curve) bandhead energies across each other at $A=242$, as seen in Fig. 1. This suggests a spontaneous breaking of the axial-octupole symmetry around this mass number. More precisely, the dominating component of octupole correlation changes from the axial $\mathrm{Y}_{30}$ at $A<242$ to the nonaxial $\mathrm{Y}_{32}$ at $A>242$. In studies of the nuclear reflection asymmetry in actinide nuclei with $A<242$, one usually neglects, as a good approximation, the nonaxial-octupole deformation and adopts only the axial $\mathrm{Y}_{30}$ component in many model calculations for the low-lying alternative parity bands (see, for example, Ref. [30]). Following the same philosophy, one should apply the nonaxial-octupole $\mathrm{Y}_{32}$ deformed mean field as a useful starting point to model the low-lying $2^{-}$bands in transfermium and superheavy nuclei, where the axial-octupole $\mathrm{Y}_{30}$ deformation component can be neglected as a good approximation. Of course, the quadrupole deformation that is superposed with octupole deformation must be included to describe the experimental moments of inertia in both actinide and transfermium nuclei.

In an effective treatment with separable forces, the octupole effect is described by the long-range octupole-octupole $(O$ - 
$O$ ) interactions between nucleons, which are produced by the matrix elements of $Y_{3 \mu}$ between single-particle states with $\Delta j=\Delta l=3$ ( $j$ and $l$ are the total angular momentum and orbit angular momentum of single-particles, respectively). The condition is found satisfied in several mass regions in the nuclear chart, and particularly in those transitional regions between spherical and well-deformed shapes. Thus, if the Fermi surface of a nucleus lies close to a pair of orbitals with $\Delta j=\Delta l=3$, the maximum octupole effect is expected. To see whether octupole correlations are present in the heavy mass region, we plot in Fig. 2 the deformed single-particle states calculated with the Nilsson potential. We note that this proton single-particle diagram created by using the Nilsson parameters of Bengtsson and Ragnarsson [31] is very similar to the diagram calculated with the Wood-Saxon potential [23]. The latter diagram has recently been used to assign experimental band structure in the odd-proton nucleus ${ }^{249} \mathrm{Bk}$ [32]. In the left part of Fig. 2, proton Nilsson diagram is plotted as a function of the quadrupole deformation $\varepsilon_{2}$. The calculation predicts a sizable spherical energy gap at proton number 114. It is particularly notable that below the gap, the positive-parity state $i_{13 / 2}$ (with $l=6$ and $j=13 / 2$ ) and the negative-parity one $f_{7 / 2}$ (with $l=3$ and $j=7 / 2$ ) appear to be nearly degenerate at the spherical limit. These two nearly-degenerate states fulfil precisely the $\Delta j=\Delta l=3$ condition for the octupole correlation. We note that the near-degeneracy of the proton $i_{13 / 2}$ and $f_{7 / 2}$ states is also a result of the Wood-Saxon single-particle diagram [23].

A careful inspection on the left part of Fig. 2 leads to another intersting discovery: When going away from the spherical limit and moving to the deformed region, one can clearly see the pairs of orbitals with opposite parities that are very close in energy and stay nearly parallel as $\varepsilon_{2}$ varies. These are the pairs $\{[633] 7 / 2,[521] 3 / 2\}$ and $\{[624] 9 / 2,[512] 5 / 2\}$. The two orbitals in each pair satisfy the $\Delta j=\Delta l=3$ condition, and in addition, are governed by $\Delta K=2$ ( $K$ is the projection of $j$ on the symmetry axis of a deformed nucleus). Thus these orbitals are connected by large matrix elements of $Y_{32}$, implying a strong triaxial-octupole correlation. The nuclei, whose proton Fermi surfaces fall in the proximity of these orbitals, may exhibit an $\varepsilon_{32}$ shape. The inserted figure at the upperright corner of Fig.2 illustrates the most favorable shape of a nucleus with triaxial-octupole deformation superposed with quadrupole deformation. Due to the strong octupole correlation the collective negative-parity states (in this case the sequence with the bandhead $I^{\pi}=2^{-}$) can be pushed down significantly.

It may possibly be true that the strong $Y_{32}$ correlation, with the occurrence of very low-lying $2^{-}$bands, is a special property in superheavy nuclei. The strict conditions for this to happen are the presence of nearly-degenerate pair(s) of singleparticle states at $\varepsilon_{2}=0$ that differ in the quantum numbers $l$ and $j$ by 3 , and due to this degeneracy at the spherical limit, the presence of closely-lying, opposite-parity, sub-orbitals for $\varepsilon_{2}>0$ that differ in the quantum number $K$ by 2 .

In the right part of Fig. 2, the diagram is obtained by includ- ing an additional $Y_{32}$ term in the Nilsson Hamiltonian varying with the octupole deformation parameter $\varepsilon_{32}$, while the quadrupole deformation is kept at $\varepsilon_{2}=0.24$. As expected, the two $\Delta K=2$ pairs feel a strong $Y_{32}$ interaction so that the force causes large energy splittings in the diagram. As a consequence, an energy gap at proton number 98 , and a one at 106 , show up. This result implies that nuclei in the mass250 region, with proton number from 96 to 108 , will possibly exhibit a strong triaxial-octupole effect with the $\mathrm{Y}_{32}$-shape. Particularly, isotopes of the element 98 (Californium) and 106 (Seaborgium) can feel very strong nonaxial-octupole correlation. However, much less $\mathrm{Y}_{32}$ effect is observed around $\mathrm{N}=150$ in the neutron single-particle diagram.

It will be more convincing if the above arguments at the mean-field level are supported by some quantitative calculations. If the observed $2^{-}$bands are indeed a manifestation of the triaxial-octupole correlation, the calculations that properly include the octupole degree of freedom should be able to reproduce them. To demonstrate this, we calculate the low-lying spectrum in these nuclei by using the RASM [33]. The RASM extends the Projected Shell Model [34] applicability by breaking the reflection symmetry in the basis and carrying out parity projection in addition to angular momentum projection. In this way, the octupole correlation is efficiently described and the model is thus particularly suitable to be applied to nuclei that show octupole effects. The trail wave function of the triaxial RASM is written in terms of parity- and angularmomentum-projected multi-qp states

$$
\left|\Psi_{I p M}\right\rangle=\sum_{K \kappa} f_{K \kappa}^{I p} \hat{P}^{p} \hat{P}_{M K_{\kappa}}^{I}\left|\phi_{\kappa}\right\rangle,
$$

where the index $\kappa$ labels deformed basis states, and $\hat{P}^{p}$ and $\hat{P}_{M K}^{I}$ are the parity-projection operator and angularmomentum-projection operator, respectively. For even-even nuclei, $\left|\phi_{\kappa}\right\rangle$ in Eq. (1) is

$$
\left\{|0\rangle, a_{v}^{\dagger} a_{v}^{\dagger}|0\rangle, a_{\pi}^{\dagger} a_{\pi}^{\dagger}|0\rangle, \cdots\right\}
$$

where $a_{v}^{\dagger}$ and $a_{\pi}^{\dagger}$ are the qp creation operator for neutrons and protons, respectively, with respect to the qp vacuum $|0\rangle$. The qp states are obtained from a deformed Nilsson calculation followed by a BCS calculation, in a model space with three major shells for each kind of nucleon (major shells 5, 6, 7 for neutrons and 4, 5, 6 for protons). The deformed Nilsson calculation is performed with the quadrupole term and the octupole term; The former term breaks the rotational symmetry, and the later the reflection symmetry, in the qp states. The utility of symmetry breaking is to introduce efficiently the corresponding correlations in the basis states. The symmetries thus spontaneously broken are recovered in the total wave function by projection (see Eq. (1)).

The two-body Hamiltonian has the form

$$
\hat{H}=\hat{H}_{0}-\frac{1}{2} \sum_{\lambda=2}^{3} \chi_{\lambda} \sum_{\mu=-\lambda}^{\lambda} \hat{Q}_{\lambda \mu}^{\dagger} \hat{Q}_{\lambda \mu}-G_{0} \hat{P}_{00}^{\dagger} \hat{P}_{00}-G_{2} \sum_{\mu=-2}^{2} \hat{P}_{2 \mu}^{\dagger} \hat{P}_{2 \mu}
$$


In Eq. (3), $\hat{H}_{0}$ is the spherical single particle Hamiltonian, the second term contains the quadrupole-quadrupole and octupole-octupole interactions, and the third and the last term are the monopole pairing and quadrupole pairing force, respectively. The interaction strengths in the second term are related to the deformation parameters through self-consistent relations

$$
\begin{aligned}
\chi_{2, \tau \tau^{\prime}} & =\sqrt{\frac{4 \pi}{5}} \frac{\frac{2}{3} \varepsilon_{2} \hbar \omega_{\tau} \hbar \omega_{\tau^{\prime}}}{\hbar \omega_{n}\left\langle Q_{20}\right\rangle_{n}+\hbar \omega_{p}\left\langle Q_{20}\right\rangle_{p}} \\
\chi_{3, \tau \tau^{\prime}} & =\sqrt{\frac{\pi}{7}} \frac{\varepsilon_{32} \hbar \omega_{\tau} \hbar \omega_{\tau^{\prime}}}{\hbar \omega_{n}\left\langle Q_{32}\right\rangle_{n}+\hbar \omega_{p}\left\langle Q_{32}\right\rangle_{p}}
\end{aligned}
$$

Therefore, for given deformations, $\chi$ are calculated, and thus not regarded as parameters. In the present work, we fix $\varepsilon_{2}=0.235$ for all nuclei; this value is verified to be consistent with that obtained from the TRS calculations. The octupole deformation parameter $\varepsilon_{32}$ influences sensitively the energy separation of the $2^{-}$band from the ground state. We find that to reproduce data, the best suitable values are: $\varepsilon_{32}=0.105$ for ${ }^{246} \mathrm{Cm}, 0.118$ for ${ }^{248} \mathrm{Cf}, 0.110$ for ${ }^{250} \mathrm{Fm}$, and 0.107 for ${ }^{252}$ No. We note that ${ }^{248} \mathrm{Cf}$ with $Z=98$ requires the largest $\varepsilon_{32}$ among the isotones. The monopole pairing strength $G_{0}$ takes the usual form

$$
G_{0}=\frac{g_{1} \mp g_{2} \frac{N-Z}{A}}{A},
$$

where the minus (plus) sign stands for neutrons (protons), and $g_{1}=20.36$ and $g_{2}=11.26$ are fixed for all the nuclei studied in this paper. The quadrupole pairing strength $G_{2}$ is assumed to be proportional to $G_{0}$, with the proportional constant 0.13 .

Diagonalizing the Hamiltonian (3) in the space spanned by parity- and angular-momentum-projected multi-qp configurations (2), we obtain a set of energy levels. From them, we plot in Fig. 3 the ground band (the lowest $\Delta I=2$ rotational sequence with positive-parity) and the $2^{-}$band (the $\Delta I=1$ sequence with negative-parity), both of which are compared with available data. As one can see in Fig. 3, agreement between theory and experiment is excellent. We also obtain a good agreement with the unpublished $2^{-}$band in ${ }^{250} \mathrm{Fm} \mathrm{[35].}$ A careful look indicates that, to compare with the neighboring isotones, the nucleus ${ }^{248} \mathrm{Cf}$ has a notably low $2^{-}$band. It is remarkable that this low $2^{-}$band can be well reproduced by using an enhanced $\varepsilon_{32}$ in the calculation (see the employed $\varepsilon_{32}$ values in the last paragraph), reflecting the strongest triaxialoctupole correlation in this nucleus. The physics behind can be understood by looking at the right part of Fig. 2. It can be clearly seen that there is an energy gap at the proton number 98. The emergence of a gap is a consequence of the maximum triaxial-octupole interaction, and therefore, ${ }^{248} \mathrm{Cf}$ shows the lowest $2^{-}$band. For the same reason, we expect very low energy $2^{-}$band in the $\mathrm{Sg}$ nuclei (the element 106).

These low-lying $2^{-}$bands are collective in nature. The degree of octupole collectivity is generally determined by an enhancement of reduced $E 3$ transition probability $B(E 3)$ over the single-particle estimate. The reduced rate of electromagnatic transitions between the eigenstates $\left|\psi_{I p M}\right\rangle$, induced by a spherical tensor operator $\hat{M}_{\lambda \mu}$, is

$$
B(M \lambda ; i \rightarrow f)=\frac{2 I_{f}+1}{2 I_{i}+1}\left|\left\langle\Psi_{I_{f} \pi_{f}}^{\sigma_{f}}\left\|\hat{M}_{\lambda}\right\| \Psi_{I_{i} \pi_{i}}^{\sigma_{i}}\right\rangle\right|^{2}
$$

and the reduced matrix elements become

$$
\begin{aligned}
& \left\langle\Psi_{I_{f} \pi_{f}}^{\sigma_{f}}\left\|\hat{M}_{\lambda}\right\| \Psi_{I_{i} \pi_{i}}^{\sigma_{i}}\right\rangle=\frac{1}{2}\left(1+\pi_{f} \pi_{i} \pi_{\lambda}\right) \\
& \sum_{K_{i} \kappa_{i} K_{f} \kappa_{f}} f_{K_{f} \kappa_{f}}^{\sigma_{f} I_{f} \pi_{f}} f_{K_{i} \kappa_{i}}^{\sigma_{i} I_{i} \pi_{i}} \sum_{\mu}\left\langle I_{i} K_{f}-\mu \lambda \mu \mid I_{f} K_{f}\right\rangle \\
& \left\langle\Phi_{\kappa_{f}}\left|\hat{M}_{\lambda \mu} P_{K_{f}-\mu K_{i}}^{I_{i} \pi_{i}}\right| \Phi_{\kappa_{i}}\right\rangle .
\end{aligned}
$$

The calculated reduced transition probabilities from ground state $0^{+}$to the $3^{-}$state of the $2^{-}$band, $B\left(E 3 ; 0^{+} \rightarrow 3^{-}\right)$, are shown in Fig. 3 in Weisskopf (single-particle) units (W.u.) (see the values marked by ' $x$ '). Again, the most enhanced triaxial-octupole collectivity appears in ${ }^{248} \mathrm{Cf}$, where the $B(E 3)$ value is the largest.

As we are exploring a completely unknown island of superheavy elements, it is very useful to understand the structure of the mass-250 nuclei, which are the gateway to the superheavy region. Most studies on the excited configurations to date have been focused on the multi-quasiparticle structure (see, for example, Refs. 36, 37]). Recently, alternative parity bands in the heaviest nuclei are predicted [38]. The excited configurations are sensitive to the detailed shell structure, and thus can bring more useful information. The degeneracy of the proton $i_{13 / 2}$ and $f_{7 / 2}$ states found in the single-particle diagram may be accidental; however, it creates the right condition to form the strong triaxial-octupole correlation that explains the experimental data. Thus, the present proposal seems to set up a new, strong constraint on all mean field calculations that are employed to predict shell structure for the superheavy mass region.

In conclusion, the nonaxial-octupole $Y_{32}$ correlation has been found to be important in the transfermium and superheavy region for the elements from 96 to 108, with the strongest effect in the elements 98 and 106. We have demonstrated that if this correlation is considered in realistic RASM calculations, the experimentally observed low-energy $2^{-}$bands in the $N=150$ isotonic chain can be easily described. The present work has thus suggested that this exotic nuclear effect, which has been discussed theoretically in other mass regions but with no convincing experimental evidences, may show up in the heaviest nuclei. The unique role of the nonaxial-octupole degrees of freedom gives weight to the recent prediction of tetrahedral symmetry in atomic nuclei. It has also set up a constraint on theoretical single-particle states for the superheavy region. The reduced E3 transition probabilities for the Coulomb excitation from the $0^{+}$ground states to the $3^{-}$states of the $2^{-}$bands are predicted to be strong. The future measurements of E3 transition rates would be crucial for the final determination of the nonaxial octuple nature 


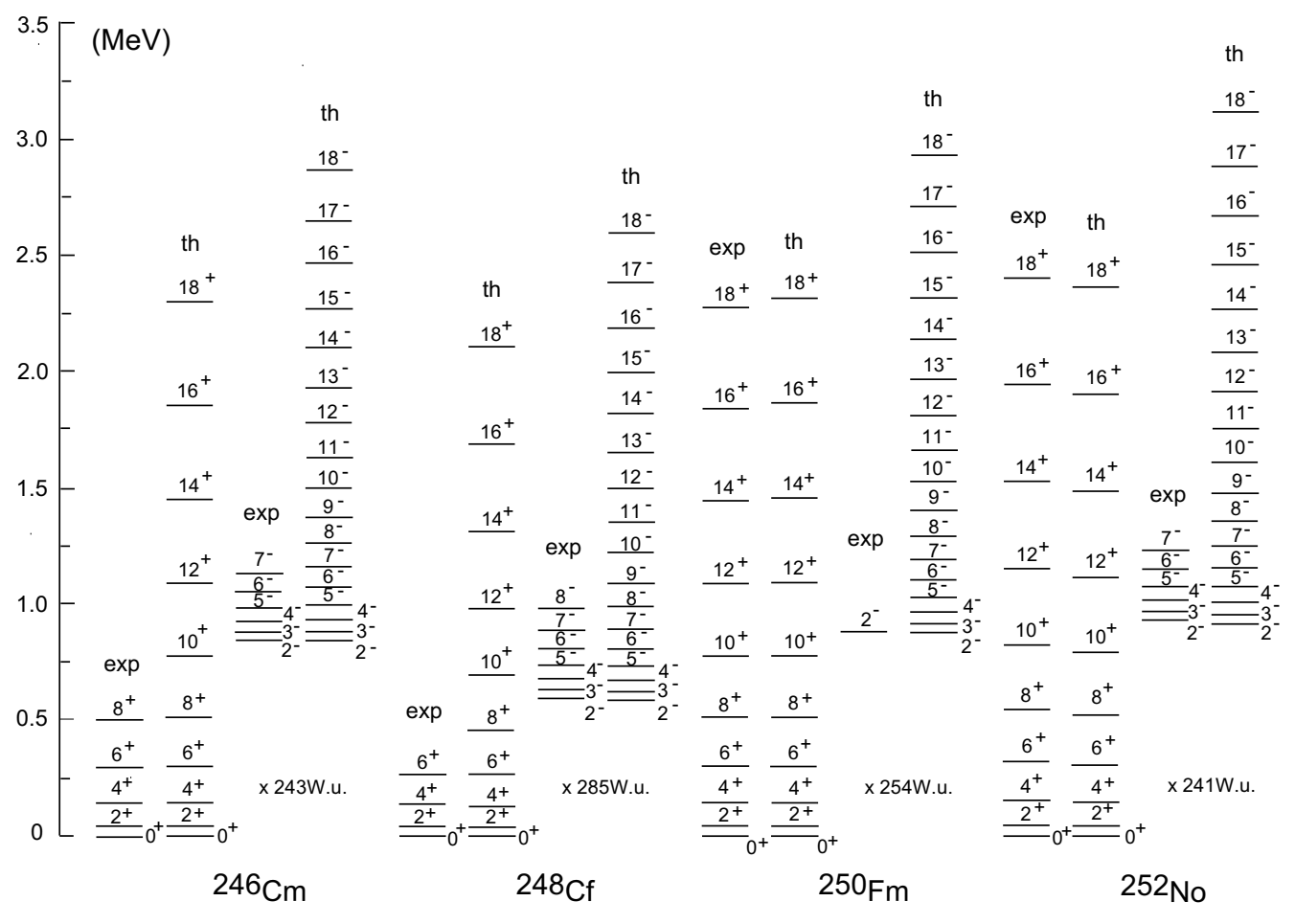

FIG. 3: The RASM calculations for the ground band and $2^{-}$band in the $N=150$ isotones. The theoretical results are compared with available data. Note the very low $2^{-}$band in ${ }^{248} \mathrm{Cf}$. The calculated $\mathrm{B}\left(\mathrm{E} 3 ; 0^{+} \rightarrow 3^{-}\right)$value in Weisskopf unit is marked by ' $\mathrm{x}$ '.

of the $2^{-}$bands and, consequently, their relation to the tetrahedral symmetry.

The authors thank R.-D. Herzberg and P. T. Greenlees for sharing the data prior to publication, and P. M. Walker for stimulating discussions. This work is supported by NNSF of China under contract No. 10475115, 10305019, 10435010, by the Chinese Major State Basic Research Development Program through grant 2007CB815005, and by the the U. S. National Science Foundation through grant PHY-0216783.

[1] I. Hamamoto, B. Mottelson, H. Xie, and X. Z. Zhang, Z. Phys. D 21, 163 (1991).

[2] S. Takami, K. Yabana and M. Matsuo, Phys. Lett. B431, 242 (1998).

[3] J. Dudek, A. Gozdz, N. Schunck and M. Miskiewicz, Phys. Rev. Lett. 88, 252502(2002).

[4] N. Schunck, J. Dudek, A. Gozdz, and P. Regan, Phys. Rev. C69, 061305(R) (2004).

[5] J. Dudek, D. Curien, N. Dubray, J. Dobaczewski, V. Pangon, P. Olbratowski, and N. Schunck, Phys. Rev. Lett. 97, 072501 (2006).

[6] A. Bohr and B. R. Mottelson, Nuclear Structure, Vol. II (W.A. Benjamin, Inc., New York, 1975).

[7] J. Dudek, A. Gozdz and N. Schunck, Acta. Phys. Pol. B34, 2491 (2003).

[8] K. Zberecki, P. Magierski, P.-H. Heenen, and N. Schunck, Phys. Rev. C 74, 051302(R) (2006).

[9] F. Frisk et al., Phys. Scr. 50, 628 (1994).
[10] R. R. Chasman and I. Ahmad, Phys. Lett. B 182, 261 (1986).

[11] P. Butler and W. Nazarewicz, Rev. Mod. Phys. 68, 349 (1996).

[12] T. Nakatsukasa, K. Matsuyanagi, S. Mizutori, and Y. R. Shimizu, Phys. Rev. C 53, 2213 (1996).

[13] M. Yamagami, RIKEN REV, VOL. 39, 78 (2001).

[14] P. Olbratowski, J. Dobaczewski, P. Powalowski, M. Sadziak, K. Zberecki, Int. J. Mod. Phys. E15, 333(2006).

[15] I. Ahmad and P. A. Butler, Annu. Rev. Nucl. Part. Sci. 43, 71 (1993).

[16] S. Hofmann and G. Münzenberg, Rev. Mod. Phys. 72, 733 (2000).

[17] P. Armbruster, Annu. Rev. Nucl. Part. Sci 50, 411 (2000) .

[18] S. Hofmann, Prog. Part. Nucl. Phys. 46, 293 (2001) .

[19] Yu. Ts. Oganessian, Nucl. Phys. A 787, 343c (2007) .

[20] M. Leino and F. P. Heßberger, Annu. Rev. Nucl. Part. Sci. 54 175 (2004).

[21] R.-D. Herzberg, J. Phys. G 30, R123 (2004).

[22] P. T. Greenlees, Nucl. Phys. A 787, 507c (2007).

[23] R. R. Chasman, I. Ahmad, A. M. Friedman, and J. R. Erskine, Rev. Mod. Phys. 49, 833(1977).

[24] P. C. Sood, D. M. Headly, R. K. Sheline, At. Data Nucl. Data Tables 51, 273 (1992).

[25] G. Hackman et al., Phys. Rev. C 57, R1056 (1998).

[26] A. Artna-Cohen, Nucl. Data Sheets 84, 901 (1998).

[27] Y. A. Akoyali, Nucl. Data Sheets 87, 249 (1999).

[28] A. Pritchard, Ph.D. thesis, Liverpool 2006.

[29] B. Sulignano et al., Eur. Phys. J. A 33, 327 (2007).

[30] G. A. Leander and Y. S. Chen, Phys. Rev. C 37, 2744 (1988).

[31] T. Bengtsson and I. Ragnarsson, Nucl. Phys. A 436, 14 (1985).

[32] I. Ahmad, et al., Phys. Rev. C 71, 054305 (2005).

[33] Y. S. Chen and Z. C. Gao, Phys. Rev. C 63014314 (2000).

[34] K. Hara and Y. Sun, Int. J. Mod. Phys. E 4, 637 (1995). 
[35] P. T. Greenlees, private communication.

[36] R.-D. Herzberg, et al., Nature 442, 896 (2006).

[37] S. K. Tandel, et al., Phys. Rev. Lett. 97, 082502 (2006).
[38] T. M. Shneidman, G. G. Adamian, N. V. Antonenko, and R. V. Jolos, Phys. Rev. C 74, 034316 (2006). 